\title{
LANDSAT 9 THERMAL INFRARED SENSOR 2 PRELIMINARY STRAY LIGHT ASSESSMENT
}

\author{
Matthew Montanaro $^{1}$, Joel McCorkel ${ }^{2}$, June Tveekrem ${ }^{2}$, John Stauder ${ }^{3}$, \\ Allen Lunsford ${ }^{4}$, Eric Mentzell ${ }^{2}$, Jason Hair ${ }^{2}$, Dennis Reuter ${ }^{2}$ \\ ${ }^{1}$ Rochester Institute of Technology, Rochester, NY 14623, USA \\ 2 NASA Goddard Space Flight Center, Greenbelt, MD 20771, USA \\ ${ }^{3}$ Utah State University, North Logan, UT 84341, USA \\ ${ }^{4}$ Catholic University of America, Washington, DC 20064, USA
}

\begin{abstract}
Although the Thermal Infrared Sensor 2 (TIRS-2) is a nearidentical copy of the Landsat 8/TIRS-1 instrument, an important design change to the optical system was designed to mitigate the stray light issue that plagued the TIRS-1 instrument $[1,2,3]$. This change involved the addition of several baffles strategically placed within the optical telescope to block the stray light paths that were present in the TIRS1 design. The specific optical changes were determined by first characterizing the TIRS-1 stray light paths on-orbit and then deriving a detailed optical model that was used to determine the locations and shapes of the mitigating baffles. The stray light design changes to the TIRS-2 instrument were confirmed through the initial thermal-vacuum characterization tests. Preliminary assessments of TIRS-2 indicate that the total stray light magnitude has been drastically reduced to a total magnitude of approximately $1 \%$ or less.
\end{abstract}

Index Terms- Landsat 9, TIRS-2, prelaunch characterization, stray light, scattered light

\section{INTRODUCTION}

The Thermal Infrared Sensor 2 (TIRS-2) payload for the Landsat 9 mission closely follows the design of the TIRS-1 instrument currently flying aboard Landsat $8[1,2]$. Shortly after launch, stray light artifacts were observed in Earth imagery from TIRS- 1 up to magnitudes of $4 \%$ (10.8 $\mu \mathrm{m}$ band) and $8 \%$ (12.0 $\mu \mathrm{m}$ band) [3]. Early on-orbit assessments utilized the Moon as a concentrated out-of-field source to map out the angles from which out-of-field radiance was detected on the focal plane arrays. The lunar scans yielded a preliminary map of stray light source angles with the primary stray light signal eminating from approximately $13^{\circ}$ off-axis (well beyond the $\pm 7.5^{\circ}$ field-of-view of the instrument) [3]. Reverse modeling of the lunar data determined that the primary stray light signal was due to higher than predicted scattering from the mechanical bracket that secures the third lens in the telescope. Out-of-field radiance directly scatters off this bracket and onto the focal plane arrays which manifests itself as extra signal on each detector. A software fix has since been implemented in the Landsat image processing system that greatly reduces the image artifacts due to the stray light for TIRS-1 [4]. However a strong effort was made to implement a hardware fix to the TIRS-2 design to prevent a similar stray light problem with the new instrument. This document will discuss the optical modeling used to derive a hardware modification of the optical system and discuss the stray light measurements from the initial thermal-vacuum (TVAC) chacterization test of the TIRS-2 instrument.

\section{OPTICAL MODEL AND STRAY LIGHT MITIGATION}

Optical models of the TIRS-2 design were assembled by two independent groups at the Space Dynamics Laboratory at Utah State University and at NASA Goddard Space Flight Center. Both groups utilized computer aided design (CAD) models to inform the physical placement of all the optical surfaces along with library material properties for each surface. These data were input to the Zemax optical modeling software to map ray traces through the optical system. Additionally, an optical model of the calibration ground support equipment (CGSE), used to measure stray light from the instrument in thermal-vacuum conditions, was produced to characterize any expected scattered light signal from the CGSE itself.

The optical modeling determined that a series of strategically placed baffles in the TIRS- 2 optical system would effectively cut off the most offending stray light source paths reaching the detectors. As illustrated in Fig. 1, the optical system consists of four refractive lenses held in place by a series of mechanical brackets and surrounded by thermal isolation shells. The telescope focuses incoming radiance onto a focal plane that consists of spectral interference filters mounted onto three detector arrays [2]. Ray tracing analyses has in- 


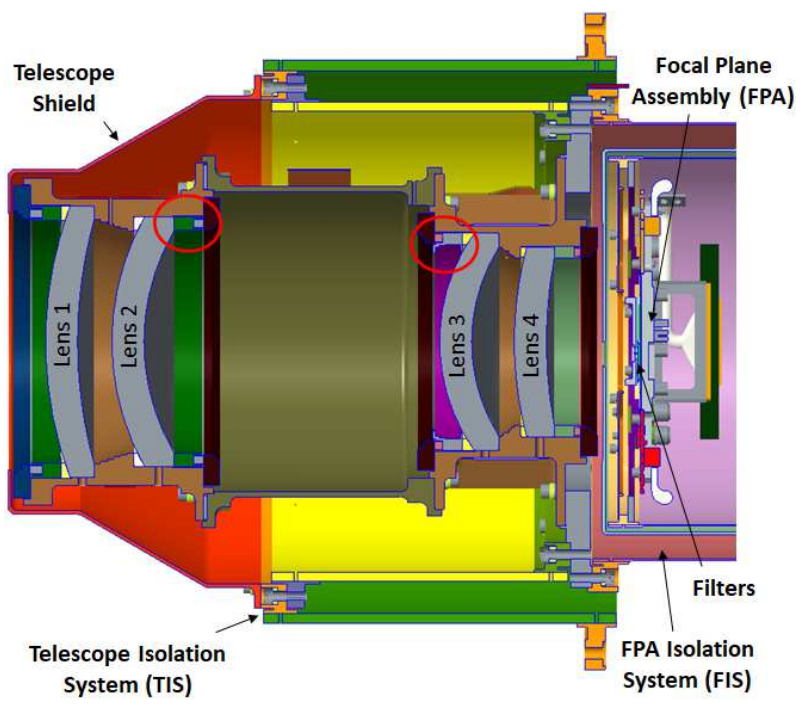

Fig. 1. Cross-section of the TIRS-2 optical system illustrating the location of the four refractive lenses, focal plane, and mechanical structure. The two red circles near Lens 2 and 3 indicate the location of the added stray light baffles.

dicated that baffles inserted at the Lens 2 and Lens 3 locations would be effective at drastically reducing the magnitude of scattered light from the mechanical surfaces in the optical system while keeping any vignetting effects to a minimum. These baffle locations are marked by the two red circles in Fig. 1.

The baffles at the Lens 3 location would address the primary scattered light paths (at $13^{\circ}$ off-axis) while the baffles at the Lens 2 location were also added since the optical modeling indicated a possible scattered light signal from $22^{\circ}$ offaxis under certain scenarios. Although the on-orbit observations of the $22^{\circ}$ scattering from TIRS- 1 is only slightly above the noise level, it was decided to also include the Lens 2 baffle as a risk mitigation. The optical model indicated the baffle would reduce the scattering effect at $22^{\circ}$ to $1 / 3$ of the signal if no baffle was added.

The optical model and baffle design were verified in a laboratory setting by illuminating a spare flight telescope by a collimated long-wave infrared source. The geometry of the set up was modified to step the incoming source signal through the out-of-field angles up to approximately $30^{\circ}$ offaxis to encompass the known stray light signals at $13^{\circ}$ and $22^{\circ}$. Figure 2 illustrates the resulting signals from the optical model (dotted lines) and the laboratory measurements (solid lines). The measurements were obtained both with and without the new baffles installed. As indicated in the figure, a reduction in scattered signal of greater than an order of magnitude could be expected by the installation of the baffles. Additionally, laboratory measurements matched the results of the optical models very closely, providing confidence in the optical models and in the baffle design.

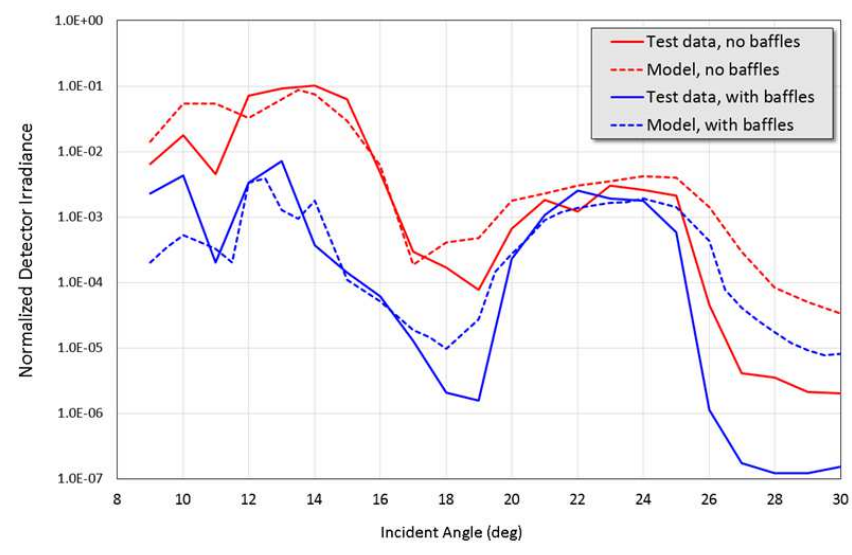

Fig. 2. Stray light signal as a function of the off-axis angle of the source. Red lines indicate the signal without baffles and blue lines indicate the signal with baffles installed. The dotted lines are the signals predicted from the optical model while the solid lines are measured signals from a laboratory set up of a flight spare telescope. These data demonstrate the effectiveness of the added baffles at reducing the scattered signal.

\section{PRELIMINARY TVAC STRAY LIGHT CHARACTERIZATION RESULTS}

The TIRS-2 instrument is required to operate at cryogenic temperatures (approximately 190K for the optics and 40K for the focal plane) to effectively measure thermal infrared radiance from the Earth [2]. The optical modeling provided the means to identify the cause and solution to the observed stray light issues. The laboratory measurements provided strong evidence that the optical models are correct and the new baffles would be sufficient in reducing the scattered light in the optical system. However, the ultimate confirmation must come with the instrument in a flight-like configuration which can only be done under thermal-vacuum conditions.

The initial TVAC campaign was known as the TIRS-2 Imaging Performance and Cryoshell Evalution (TIPCE) and was performed at the NASA Goddard Space Flight Center [5]. The instrument configuration consisted of the major components of the optical and detector systems including the complete telescope, focal plane with mounted filters, and thermal isolation shells. Although the complete instrument structure and scene select mirror was not installed, a series of mechanical apertures collectively known as the front end baffle simulator (FEBSim) was mounted in front of the telescope to simulate the entrance aperture of TIRS-2. The CGSE contains a variable temperature and variable aperture blackbody source that can be positioned at various locations in the TIRS-2 infield and out-of-field by the use of a steering mirror in the CGSE. This configuration allowed for the CGSE source to be positioned between $-28^{\circ}$ to $+18^{\circ}$ in the azimuth (across-track) 


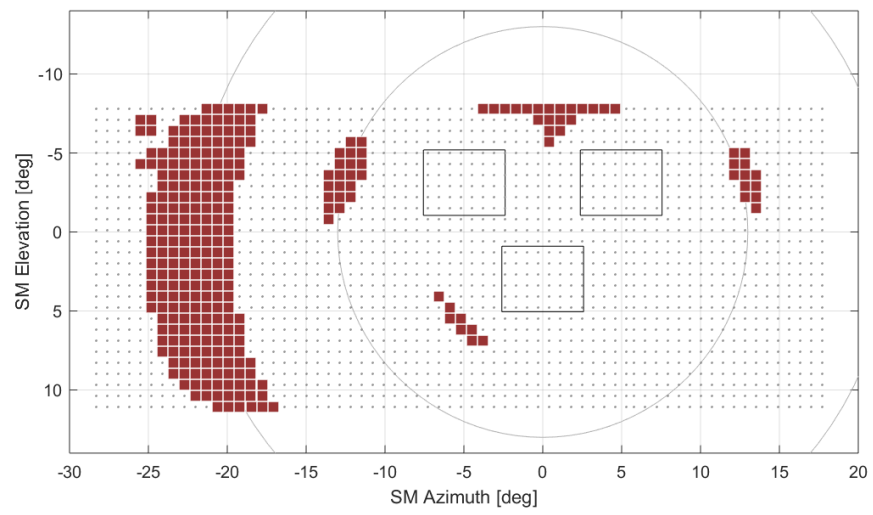

Fig. 3. Grid locations of the CGSE 0.7-degree source relative to the TIRS-2 optical axis. Each dot indicates the center of the source. Source locations where scattering was observed anywhere on the arrays are inidcated by the red grid locations. The three rectangles near the center of the optical axis indicate the position of the three focal plane arrays, the inner circle illustrates the $13^{\circ}$ scattering annulus, and the outer circle illustrates the $22^{\circ}$ scattering annulus.

direction and between $-8^{\circ}$ to $+12^{\circ}$ in the elevation (alongtrack) direction. At each source location the resulting signal on the focal plane detectors can be recorded. Although the entire out-of-field area could not be scanned due to the physical limitations of the CGSE, the angles that were able to be scanned were sufficient to envelop the azimuthal extent of the residual scattered light sources and provide an initial assessment of whether the added baffles resulted in the expected reduction of the scattered light signals.

TIRS-2 is required to be sensitive to source signals between $240 \mathrm{~K}$ and $360 \mathrm{~K}$ without saturating. To facilitate this, the integration time is usually set to $2.4 \mathrm{~ms}$ so that an observed blackbody source of $360 \mathrm{~K}$ does not saturate. However, to make the instrument as sensitive as possible for the TIPCE stray light measurements, the CGSE source was raised to its maximum of $500 \mathrm{~K}$ and the detector integration time was raised to its maximum value of $5.5 \mathrm{~ms}$. This combination would saturate the detectors when the CGSE source is viewed directly but produces an enhanced out-of-field signal for stray light measurements. Any observed stray light signals were scaled to a percentage by determining the unsaturated directly-illuminated source signal. For the TIPCE stray light measurements, the CGSE source with a 0.7 degree square aperture was positioned at grid locations spanning $-28^{\circ}$ to $+18^{\circ}$ in the azimuth direction and $-8^{\circ}$ to $+12^{\circ}$ in the elevation direction (see Fig 3). At each grid location, ten frames of the source were acquired followed by ten frames of the background (shutter). The mean of the background was subtracted from the mean of the source for each grid location and then scaled to a percentage of the directly illuminated source signal.

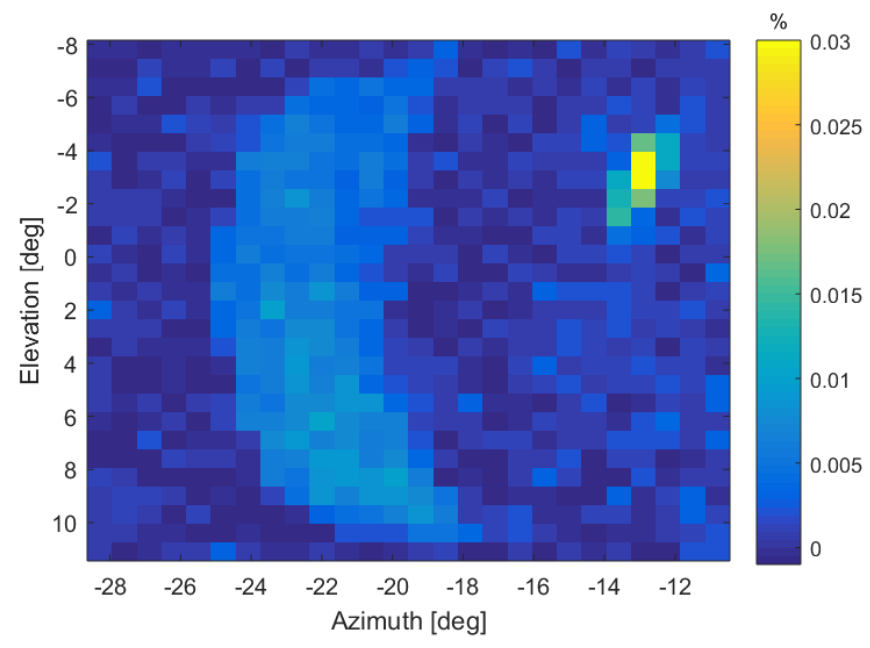

Fig. 4. Map illustrating the magnitude of the scattered signal detected on the $12 \mu \mathrm{m}$ band when the source was at each grid location on the left side of the focal plane. The scattering signal is mostly noise except for a small feature located at $13^{\circ}$ in azimuth and a broader, weaker signal located at $22^{\circ}$ in azimuth. These features correspond to the residual stray light signal from the baffles at Lens 3 and Lens 2, respectively. The scattering magnitude is approximately $0.03 \%$ when the source was at $13^{\circ}$ and the magnitude is approximately $0.01 \%$ when the source was at $22^{\circ}$.

The grid location associated with scattered signal observed on any detector array was flagged as illustrated in Fig 3. For clarity, only signals that were obviously caused by optical scattering were flagged in the figure. Signals that were traced to direct illumination by the CGSE source were omitted. Although the TIPCE measurements (i.e.- grid locations) are not perfectly symmetric on either side of the focal plane arrays, it is expected that the recorded scattering signals due to sources on the left side of the focal plane would be mirrored by sources on the right side of the focal plane due to the cylindrical symmetry of the optical system. In other words, the detected scattering at $22^{\circ}$ on the left side of the focal plane is expected to be mirrored at $22^{\circ}$ on the right side of the focal plane even though those right-hand angles were unable to be scanned during the TIPCE data collection. To illustrate the magnitudes of the detected scattering, a map was produced in Fig 4 of the left-side out-of-field area in which each pixel represents a scanned grid point and its value represents the absolute magnitude of the scattering at that grid location. This map of the left side out-of-field area demonstrates the magnitude of the residual stray light signal. The small feature at $13^{\circ}$ corresponds to the residual signal from the Lens 3 baffle and has a magnitude of about $0.03 \%$. The broad feature at $22^{\circ}$ corresponds to the residual signal from the Lens 2 baffle and has a magnitude of about $0.01 \%$.

Several important observations were made from the 
TIPCE measurements. Using the TIRS-1 lunar scans as a guide, several specific out-of-field locations identified in the lunar data produced a significant scattered signal on the TIRS- 1 detectors of approximately $0.4 \%$ for out-of-field locations around $13^{\circ}$ off-axis. However, those same out-of-field locations (i.e.- grid points) were scanned during TIPCE and no scattered signal above the background was recorded. For other grid locations around $13^{\circ}$ off-axis in TIPCE, the magnitude of the recorded signal was significantly reduced to approximately the $0.03 \%$ level. These results indicate that the distribution and magnitude of the $13^{\circ}$ stray light signal (the primary scattered light source for TIRS-1) has been drastically reduced in TIRS-2 demonstrating that the new Lens 3 baffle is having the desired effect.

Residual scattering was also observed in TIRS-2 around $22^{\circ}$ off-axis as predicted by the model and weakly observed in TIRS-1. At an out-of-field source location around $22^{\circ}$, the magnitude of the scattered signal observed by TIRS-2 is approximately $0.01 \%$ compared to a signal of approximately $0.024 \%$ observed by TIRS- 1 on-orbit. This result demonstates that the new Lens 2 baffle is reducing the $22^{\circ}$ scatter to the expected $1 / 3$ of the magnitude of the TIRS- 1 scatter.

In addition to particular out-of-field locations, the summation of signal from all out-of-field angles is an important metric since the Earth fills the out-of-field area of TIRS-2 on-orbit and the total stray light signal may potentially affect the recorded direct Earth signal (as it does on TIRS-1). The summation of the recorded signal from all grid locations scanned in TIPCE is approximately $0.34 \%$ in the worst case. This summation, however, is only over the source angles that could be scanned in the TIPCE TVAC test in which most of the azimuth direction was scanned but higher angles in the elevation direction could not be scanned due to the hardware limits of the TVAC configuration. To extrapolate what the total scattered signal would be over all out-of-field angles, the optical model can be utilized along with the TIPCE measurements. The sum of the optical model over the same angles that were measured in TIPCE was calculated and then compared to the sum of the TIPCE measurements in order to derive a scaling factor between the optical model and the TIPCE measurements. This scaling factor was then applied to the entire optical model to scale the model into the same units as the TIPCE measurements. The resulting summation of the scaled optical model yields estimates of the total expected stray light magnitude for each detector array. The worst-case estimate is a $1.1 \%$ total stray light magnitude for the $12.0 \mu \mathrm{m}$ band of detector array A.

\section{SUMMARY}

The preliminary stray light assessment of the TIRS-2 instrument indicates that the stray light effects have been drastically reduced in both shape and magnitude. The scattering sources from the TIRS-1 instrument were identified by on-orbit lunar data collections and used to inform a new optical model of the TIRS-2 instrument. The optical models were used to determine a hardware correction in the form of baffles inserted into the optical system that would block the most offending scattered light paths from reaching the focal plane detectors. The effects of these baffles were first measured under ambient conditions in the laboratory and results confirmed the optical model design. Initial stray light measurements were then performed in flight-like thermal vacuum conditions with the instrument operating as it would on-orbit. Results from the TVAC measurements show that the primary scattering path from TIRS- 1 has been reduced to about $0.03 \%$ in magnitude for a signal source location at $13^{\circ}$ off-axis (down from $0.4 \%$ for the same angles for TIRS-1). Additionally, the total stray light effect in TIRS-2 is reduced significantly to about $1 \%$ or less in TIRS-2 versus an up to $8 \%$ total magnitude in the TIRS-1 design. The results from the initial TVAC testing provided added confidence in the modified optical design of the instrument. Further refined optical models are currently being produced to derive a spatially-explicit and higher fidelity estimate of the total expected scattering signal. Studies will also be performed to determine the impact on Landsat science products due to the summed stray light signal so that the added signal due to the scattering can be factored into the uncertainty analysis of the radiometric calibration of the TIRS-2 instrument.

\section{REFERENCES}

[1] D. Reuter, C. Richardson, F. Pellerano, J. Irons, R. Allen, M. Anderson, M. Jhabvala, A. Lunsford, M. Montanaro, R. Smith, Z. Tesfaye, and K. Thome, "The Thermal Infrared Sensor (TIRS) on Landsat 8: Design Overview and Pre-Launch Characterization," Remote Sensing, vol. 7, no. 1, pp. 1135-1153, 2015.

[2] J. Hair, D. Reuter, S. Tonn, A. Simon, J. McCorkel, and M. Djam, et al., "Landsat 9 Thermal Infrared Sensor 2 Architecture and Design Overview," These Proceedings.

[3] M. Montanaro, A. Gerace, A. Lunsford, and D. Reuter, "Stray Light Artifacts in Imagery from the Landsat 8 Thermal Infrared Sensor,' Remote Sensing, vol. 6, no. 11, pp. 10435-10456, 2014.

[4] A. Gerace and M. Montanaro, "Derivation and validation of the stray light correction algorithm for the Thermal Infrared Sensor onboard Landsat 8," Remote Sensing of Environment, vol. 191, pp. 246-257, 2017.

[5] J. McCorkel, M. Montanaro, B. Efremova, A. Pearlman, B. Wenny, A. Lunsford, A. Simon, J. Hair, and D. Reuter, "Landsat 9 Thermal Infrared Sensor 2 Characterization Plan Overview," These Proceedings. 